\title{
CDISC QT Studies Therapeutic Area User Guide Version 1.0
}

National Cancer Institute

\section{Source}

National Cancer Institute. CDISC QT Studies Therapeutic Area User Guide Version 1.0. NCI Thesaurus. Code C161449.

The 1.0 version of the CDISC QT Studies therapeutic area user guide. 